Revue européenne des sciences sociales

European Journal of Social Sciences

XLI-125 | 2003

Index de la Revue européenne des sciences sociales (1995-2002)

\title{
Index des noms
}

\section{(2) OpenEdition}

\section{Journals}

Édition électronique

URL : http://journals.openedition.org/ress/556

DOI : $10.4000 /$ ress.556

ISSN : 1663-4446

Éditeur

Librairie Droz

\section{Édition imprimée}

Date de publication : 1 janvier 2003

Pagination : $35-38$

ISBN : 2-600-00844-6

ISSN : 0048-8046

Référence électronique

"Index des noms », Revue européenne des sciences sociales [En ligne], XLI-125 | 2003, mis en ligne le

30 novembre 2009, consulté le 22 avril 2019. URL : http://journals.openedition.org/ress/556 ; DOI :

$10.4000 /$ ress.556 


\section{INDEX DES NOMS}

Achard Pierre 1454

Alker, Hayward 1410

Aqueci, Francesco 1224, 1255, 1499

Arliaud, Michel 1260

Ascher, Edgar 1246, 1353, 1395

Baertschi, Bernard 1482

Balandier, Georges 1299

Balle, Francis 1466

Barbichon, Guy 1554

Bassoul, René 1406

Baume, Sandrine 1569

Beetham, David 1232

Béguin, Hubert 1288, 1335, 1376

Bénéton, Philippe 1415

Berthelot, Jean-Michel 1287, 1596

Berthoud, Gérald 1298, 1309, 1361, $1435,1478,1525,1537,1572$, 1582

Besnier, Jean-Michel 1581

Bon, Frédéric 1408

Bondolfi, Alberto 1481

Borel, Marie-Jeanne 1279, 1294, 1333, 1508

Boudon, Raymond 1326,

1414, 1452, 1548

Boudot, Maurice 1419

Bourg Dominique 1526

Bouvier, Alban 1344, 1422, 1453, 1500,1587

Boyer, Alain 1417

Breton, Philippe 1574

Breuer, Stefan 1234

Bridel, Pascal 1293, 1441, 1485, 1541, 1555

Bruhns, Hinnerk 1239

Bruni, Luigino 1494

Bruttin, Françoise 1230

Busino, Giovanni 1225, 1247, 1251, $1278,1291,1305,1320,1343$, 1369, 1400, 1430, 1432, 1445,
$1507,1512,1523,1536,1552$, 1568,1600

Busino-Salzmann, Barbara 1366

Caillé, Alain 1310, 1425

Candau, Joël 1387

Cardini, Antonio 1493

Cerezuelle, Daniel 1390

Cerqui Ducret, Daniela 1348, 1396, 1572,1583

Chamboredon, Jean-Claude 1259

Chevalley, Catherine 1585

Chazel, François 1235

Claval, Paul 1451

Clément, Fabrice 1325

Coenen-Huther, Jacques 1228, 1295 , 1322, 1339, 1379, 1429, 1437. $1518,1535,1544,1565,1566$, 1595

Colliot-Thélène, Catherine 1233

Cuin, Charles-Henry 1550, 1597

Dagenais, Bernard 1458

Dagenais, Marcel G. 1442

Dagum, Camilo 1444

Davallon, Jean 1264

De Frouville, Olivier 1551

De Lara, Philippe 1426, 1479

De Singly, François 1256

Dewitte, Jacques 1468

Di Donato, Riccardo 1304

Di Nuoscio, Enzo 1524

Dion, Michel 1407

Dockès, Pierre 1377, 1486

Draus, Franciszek 1240

Dubois, Michel 1484, 1593

Dufour, Alfred 1324

Duprat, Gérard 1231

Elster, Jon 1409

Ethis, Emmanuel 1367 
Fabiani, Jean-Louis 1273, 1367, 1527

Farge, Arlette 1261

Farrell, Thomas B. 1449

Fassa, Farinaz 1572, 1580, 1594

Federici, Maria Catherina 1497

Feldman, Jacqueline 1553, 1589

Fidomanzo, Vincenzo 1571

Fillieule, Renaud 1399

Finger, Matthias 1327

Fiorini, Rafaella 1520

Fischman, Marianne 1534

Fivaz-Silbermann, Ruth 1220, 1314

Flückiger, Alexandre 1559

Fontela, Emilio 1601

Fournier, Marcel 1300

Frobert, Ludovic 1317

Galland, Blaise 1385

Gardin, Jean-Claude 1271

Gaudin, Jean-Pierre 1284, 1337, 1380, 1515,1556

Gerbner, George 1457

Gerstlé, Jacques 1456

Géhin, Etienne 1418

Glassner, Jean-Jacques 1386

Goujon, Philippe 1227, 1244, 1328

Grall, Bernard 1363

Gras, Alain 1347

Grignon, Claude 1257, 1533, 1584

Grinevald, Jacques 1352

Grize, François 1391

Grize, Jean-Blaise 1226, 1241, 1249 , $1272,1323,1360,1391,1546$

Hamel, Jacques 1229

Herzlich, Claudine 1461

Holly, Alberto 1341

Hottois, Gibert 1346

Hummel, Hans J. 1403

Hunyadi, Mark 1558

Isambert, François 1254

Ischy, Frédéric 1572, 1573

Jacob, Pierre 1586

Jamous, Haroun 1404

Jorland, Gérard 1590

Jouanjan, Olivier 1542, 1560
Jufer, Marcel 1350

Karsenti, Bruno 1306

Kaufmann, Laurence 1599

Keifitz, Nathan 1405

Keucheyan, Razmig 1598

Kirman, Alan 1443

Kitzberger, Philip 1438

Knoepfel, Peter 1338, 1381

Kremer-Marietti, Angèle 1588

Krippendorff, Klaus 1450

Kupiec, Jean-Jacques 1473

Kuran, Timur 1465

Kütler, Wolfgang 1236

Lahire, Bernard 1318, 1393, 1567

Lautman, Jacques 1274, 1411, 1421

Lazar, Judith 1382, 1448, 1459

Le Bras, Hervé 1340, 1373, 1447 , 1519

Lenclud, Gérard 1275

Lendjel, Emeric 1364, 1503, 1534

Legris, André 1505

Lepetit, Bernard 1266

Leridon, Henri 1447

Lindenberg, Daniel 1302

Livet, Pierre 1540

Lucchini, Riccardo 1250

Lukes, Steven 1301

Mahé, Alain 1312

Malson, Lucien 1262

Maniscalo, Maria Luisa 1498

Marchionatti, Roberto 1502, 1520

Maréchal, Jean-Paul 1358

Martelli, Stefano 1303

Martignoni, Graziano 1480

Martinotti, Guido 1394

Mauss, Marcel 1311

McQuail, Denis 1460

Menger, Pierre-Michel 1258

Miège, Bernard 1575

Miéville, Ariane 1278

Mignot, Xavier 1462

Misaki, Kayoko 1489

Moessinger, Pierre 135914231439

Mohr, Pierre 1332

Molino, Jean 1270, 1285, 1330, 1383 
Montandon Cléopâtre 12481321

Moor, Pierre 1279, 1292, 1440, 1514, 1528

Morand, Charles-Albert 1289

Morin, Edgar 1467, 1483

Mornati, Fiorenzo 1501

Mouchot, Claude 1282, 1516

Moulin, Raymonde 1277

Musso, Pierre 1579

Nakakubo, Kunio 1487

Neschke, Ada Babette 1296

Noelle-Neumann, Elisabeth 1455

Noseda, Veronica 1561

Olivier de Sardan, Jean-Pierre 1269

Olson, David R. 1463

Ouédraogo, Jean-Martin 1316

Paillard, Isabelle 1576

Papilloud, Christian 1545

Pasquino, Pasquale 1297

Passeron, Jean-Claude 1280, 1342, $1384,1431,1509,1529,1538$, 1549,1557

Pedler, Emmanuel 1268, 1367

Pepe, Luigi 1495

Peraya, Daniel 1397

Perrenoud, Philippe 1416

Perriault, Jacques 1392

Petit, Annie 1477

Petitat, André 1267

Petroni, Angelo M. 1222

Pharo, Patrick 1420, 1428, 1547

Pichot, André 1474

Picon, Antoine 1357

Pierret, Janine 1461

Pignon, Dominique 1345

Piuz, Anne-Marie 1362

Poirier, Jean 1388

Poitou, Jean-Pierre 1389

Pollini, Gabriele 1496

Pomian, Krysztof 1521

Potier, Jean-Pierre 1488

Pouillon, François 1308

Prokhoris, Sabine 1371, 1434, 1511

Pteroudis, Evangelos 1336
Pumain, Denise 1375, 1436, 1515, 1562

Quéré, Louis 1424

Racine, Jean-Bernard 1290, 1336, 1370, 1436, 1561

Raffestin, Claude 1243, 1283, 1349, 1374, 1446

Ragni ludovic 1505

Ragouet, Pascal 1592

Ramognino, Nicole 1591

Raynaud, Philippe 1530

Renault, Michel 1464

Revel, Jacques 1265

Rey, Jean-Noël 1327

Rogoff-Ramsoy, Nathalie 1413

Roig, Charles 1365

Rolle, Pierre 1412

Romano, Ruggiero 1223, 1315, 1368, 1522

Rossi, Pietro 1237

Schlüpmann, Klaus 1355

Schmidt, Christian 1506

Sfez, Lucien 1577

Siméon, Jean-Pierre 1245

Simioni, Olivier 1578

Stamm, Roger Alfred 1471

Steiner, Philippe 1378, 1491, 1564

Tarascio, Vincent J. 1504

Tarot, Camille 1307

Tatti, Elena 1485

Terribilini, Serge 1338, 1381

Terrier, Jean 1570

Thinès, Georges 1475

Tibon-Cornillot, Michel 1356

Timsit, Gérard 1281, 1513, 1543

Tinland, Frank 1354, 1476

Toulouse, Gérard 1531

Tricot, Claude 1319

Valade, Bernard 1402, 1427

Van Daal, Jan 1490

Varone, Frédéric 1381

Vatin, François 1363

Verret, Michel 1276 
Veyne, Paul 1252, 1263, 1277

Vincent, Jean-Marie 1238

Vinck, Dominique 1351

Vitalis, André 1398

Vogel, Peter 1470

Volken, Henri 1286 1334, 1372, 1433, 1517, 1539, 1563

Von Boletzky, Sigurd 1469
Von Wahlert, Gerd 1472

Vullierme, Jean-Louis 1532

Waswo, Richard 1242

Widmer, Charles 1221

Wunenburger, Jean-Jacques 1510

Zanni, Alberto 1492 